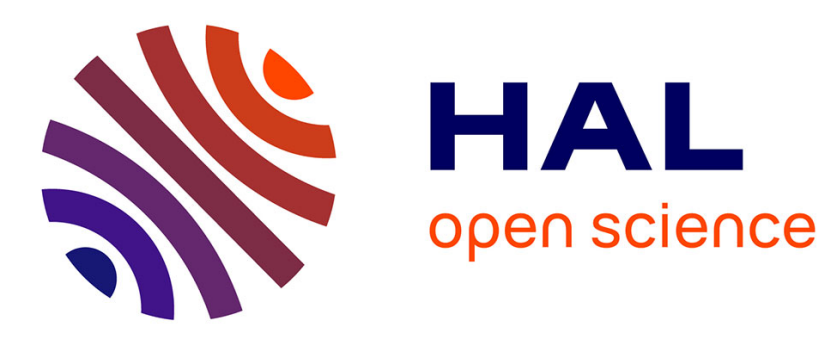

\title{
Le couteau suisse des politiques migratoires
}

Cristina del Biaggio, Ibrahim Soysüren

\section{To cite this version:}

Cristina del Biaggio, Ibrahim Soysüren. Le couteau suisse des politiques migratoires. Plein Droit, 2019, Frontières d'ailleurs, 121 (2), pp.5. 10.3917/pld.121.0005 . hal-02285116

\section{HAL Id: hal-02285116 https://hal.science/hal-02285116}

Submitted on 18 Sep 2019

HAL is a multi-disciplinary open access archive for the deposit and dissemination of scientific research documents, whether they are published or not. The documents may come from teaching and research institutions in France or abroad, or from public or private research centers.
L'archive ouverte pluridisciplinaire HAL, est destinée au dépôt et à la diffusion de documents scientifiques de niveau recherche, publiés ou non, émanant des établissements d'enseignement et de recherche français ou étrangers, des laboratoires publics ou privés. 


\section{Le couteau suisse des politiques migratoires}

Cristina Del Biaggio, géographe, université Grenoble Alpes et Laboratoire Pacte, Ibrahim Soysüren, sociologue, université de Neuchâtel

Publié dans Plein Droit, $2019 / 2$ ( $\left.\mathrm{n}^{\circ} 121\right)$, pp. 3-6.

https://www.cairn.info/revue-plein-droit-2019-2.htm

\section{Résumé :}

Le « génie suisse » a trouvé à s'exprimer dans les politiques migratoires et les instruments destinés à restreindre les arrivées de migrants, qu'ils relèvent ou non du droit d'asile. Précurseure, la Suisse reste une « source d'inspiration » en matière d'expulsion, de centres de tri, de prise d'empreintes ou de «disparition » des déboutés du droit d'asile qui en fait rêver plus d'un. Mais elle est aussi à l'origine de notions telles que celle de «pays d'origine sûr », promise à un «brillant » avenir en Europe.

Outre le couteau multifonctions rouge à croix blanche, le « génie helvétique » nous a fait don d'instruments et pratiques relevant plus de l'ingénierie sociale que de la mécanique, souvent généralisés sans bruit dans d'autres pays où elles contribuent à la mise en place de politiques migratoires restrictives. L'administration et la société civile de ces pays européens ont tout intérêt à s'intéresser de près au cas suisse pour mieux comprendre les origines et le fonctionnement de ces outils Made in Switzerland.

La liste qui suit, non exhaustive, montre la diversité des initiatives dans le domaine de l'asile, où la très fédéraliste Suisse excelle à centraliser les tâches.

\section{Politique d'asile : le centralisme au service d'une confédération}

C'est en s'inspirant du «modèle » des Pays-Bas que la Suisse a créé, dans sa dernière réforme de l'asile (2019), des « centres fédéraux » où s'effectue le dépôt de la demande d'asile ainsi que, dans $60 \%$ des cas selon les estimations officielles, l'analyse du dossier et la préparation au renvoi. Les $40 \%$ restant sont attribués à un canton où résider dans l'attente d'une réponse. Dans l'esprit de la réforme, « la priorité est [...] donnée à l'accélération des procédures aboutissant à des décisions négatives ou NEM ${ }^{1}$ Dublin ${ }^{2}$. Cette stratégie, outre l'accélération des expulsions, vise aussi à envoyer un signal dissuasif aux migrants potentiels.

Le tableau page suivante, établi à partir des données de l'Observatoire romand du droit d'asile et des étrangers (ODAE), nous permet de [fin p. 3] mieux comprendre cette priorisation des demandes selon les nationalités, qui a permis, pour certaines d'entre elles, de dépasser le cap de $100 \%$ en traitant des cas plus anciens. 


\begin{tabular}{|l|l|l|l|}
\hline Pays de provenance & Type de procédure & $\begin{array}{l}\text { Taux de traitement } \\
\text { des demandes }\end{array}$ & $\begin{array}{l}\text { Taux d'acceptation } \\
\text { en } 2013^{3}\end{array}$ \\
\hline Tunisie & Accélérée & $115 \%$ & $5 \%$ \\
\hline Nigeria & Accélérée & $113 \%$ & $16 \%$ \\
\hline Kosovo & Accélérée & $123 \%$ & $19 \%$ \\
\hline Afghanistan & Non accélérée & $70 \%$ & $91 \%$ \\
\hline Syrie & Non accélérée & $52 \%$ & $96 \%$ \\
\hline Érythrée & Non accélérée & $71 \%$ & $97 \%$ \\
\hline
\end{tabular}

La création de grands centres pose la question de l'invisibilisation des demandeurs d'asile, pour lesquels il est désormais difficile, voire impossible, d'établir des contacts avec la société suisse et les organisations de défense des migrants. Or, comme le notait déjà un rapport de $1989^{4}$, l'exécution des renvois constitue le maillon faible de la procédure d'asile : «Il arrive souvent que le canton n'exécute pas le renvoi parce que certaines personnalités ou organisations interviennent en faveur de l'étranger. " D'où l'intérêt de soumettre les demandeurs d'asile à un régime de semi-détention dans des lieux où ces «personnalités ou organisations » ne peuvent intervenir que très difficilement.

\section{« Pays sûrs » et autres trouvailles helvétiques}

La centralisation dans de grands centres, où un premier tri est opéré entre ceux placés en procédure dite normale (c'est-à-dire qui se verront très probablement reconnaître une protection) et ceux, la majorité, placés en procédure accélérée, s'est appuyée sur une autre invention : la notion de « pays sûr ». Peter Arbenz, directeur de l'Office fédéral des réfugiés dans les années 1980 et 1990, en fut le concepteur, et il s'en félicite : «Dans les consultations informelles entre pays européens, c'est la Suisse qui était à l'avant-garde. C'est nous qui avons développé l'idée de pays sûrs ».

Arbenz complète la liste : « Le système des empreintes digitales, c'était nous ; l'idée de lancer un programme d'aide au retour avec des zones de protection, les accords bilatéraux avec les pays producteurs de réfugiés, c'est encore nous », rapportent Pierre Hazan et Yaël Reinharz Hazan ${ }^{5}$. Bien plus, continuent-ils : « Édification de centres de tri, décision de non-entrée en matière, contrôle systématique des empreintes digitales et fichage des requérants, [...] procédures rapides en vue de traquer un éventuel motif de refus, mais toujours assorti de la volonté de protéger les droits des "vrais réfugiés" contre les "faux réfugiés", pression sur les fonctionnaires pour qu'ils augmentent leur productivité, instauration de mesures de contrainte permettant de détenir des candidats au refoulement, conditionnement psychologique avec menace sur l'emploi des juges de la Commission de recours en matière d'asile pour qu'ils accélèrent les cadences de leurs décisions ». On peut y ajouter les « expertises linguistiques pour vérifier les origines des migrants » en vue de leur renvoi, comme l'a déclaré Mario Gattiker, directeur du secrétariat d'État aux migrations (SEM), à la presse ${ }^{6}$. 
La liste est longue, et il faut aussi prendre en compte la capacité stratégique de maîtriser toute l'échelle décisionnelle : la mise sur pied de groupes de travail ad hoc pour analyser un problème, la transformation des recommandations en lois, et enfin leur application sur tout le territoire national.

La Suisse est aussi le premier pays au monde à avoir introduit une exception à la Convention de Genève : avec la nouvelle loi, les déserteurs ne se voient plus reconnaître la qualité de réfugié. Une mesure ad hoc pour les Érythréens, le groupe le plus important de personnes cherchant asile en Suisse (selon les statistiques officielles, sur les 62000 personnes relevant du domaine de l'asile en Suisse en 2018, 12000 étaient érythréennes).

Il fallait donc sortir la désertion des motifs d'asile. Mais aussi envoyer des représentants en Érythrée pour espérer signer un accord de réadmission qui permettrait l'exécution des renvois vers ce pays, souvent qualifié de «Corée du Nord de l'Afrique ». Les autorités helvétiques ont décidé parallèlement de réexaminer au cas par cas les quelque 3000 admissions provisoires $^{7}$ octroyées aux Érythréens à la suite d'un arrêt du 17 août 2017 par lequel le Tribunal administratif fédéral (TAF) a considéré que le retour au pays des Érythréens déboutés était en principe exigible. Ce projet de réexamen et l'arrêt du TAF ont été contestés par les associations qui défendent le droit d'asile ${ }^{8}$ et par le Comité contre la torture de l'ONU (M.G. c/Suisse, Communication $\mathrm{n}^{\circ}$ 811/2017).

D'un point de vue strictement quantitatif, cette politique de dissuasion semble fonctionner, au vu du recul du nombre de demandes d'asile des ressortissants érythréens en Suisse entre 2015 (9 966) et 2018 (2 825). Mais la courbe du nombre de dépôts des demandes d'asile des Érythréens dans les pays de l'UE montre une décroissance similaire. [fin p. 4]

En effet, le nombre de demandes d'asile déposées en Suisse ne dépend pas forcément de politiques restrictives, mais d'éléments qui lui échappent en partie, notamment les rythmes saisonniers et la conjoncture politique internationale, comme le rappelait en 2015 Vivre Ensemble dans un communiqué de presse du 29 juillet 2014. La comparaison internationale met donc à mal l'argument du recul des demandes.

\section{Dublin comme instrument de gestion des flux migratoires}

Bien avant la prétendue « crise des réfugiés », la Suisse avait déjà compris l'intérêt du système « Dublin », qui vise à empêcher les demandes d'asile multiples en Europe, comme un instrument de gestion des flux migratoires. Déjà en 1989, la Confédération s'en préoccupait, comme le signale le rapport cité plus haut : «Dans le cadre du processus d'intégration européenne, il conviendra d'accorder une attention particulière à la relation entre la Suisse et les États méditerranéens. » Sans doute parce qu'elle a dès le début considéré le règlement « Dublin » comme un instrument de régulation des flux migratoires, la Suisse a été longtemps « championne » des renvois dans ce cadre. Le personnel spécialisé dans le suivi des cas «Dublin » au niveau fédéral y est pour beaucoup. La carte des transferts nets Dublin, publiée en 2014 dans le rapport annuel de l'European Asylum Support Office (EASO) et qui faisait ressortir les renvois importants de la Suisse vers l'Italie, avait interpellé les associations de défense du droit d'asile en Suisse. 
Toutefois, le solde entre les entrées et les renvois « Dublin » commence à s'amenuiser. D'autres pays européens, l'Allemagne mais aussi la France, se sont mis à appliquer plus systématiquement le règlement "Dublin III », mais l'avantage comparatif cumulé sur plus de 10 ans par la Suisse reste non négligeable. Entre 2009 et fin octobre 2018, elle a effectué 30062 transferts « Dublin », mais n'a reçu que 6624 personnes de la part d'autres pays européens parties au règlement «Dublin »?.

La Suisse semble miser sur l'incapacité d'autres États européens de répondre aux requêtes de (re)prise qu'elle leur envoie, car ces pays deviennent responsables pour le traitement de ces cas s'ils ne s'opposent pas à ces demandes infondées dans les délais imposés par le règlement «Dublin III ». De plus, certaines de ces requêtes seraient soumises sans preuve pertinente, alors que ce texte européen exige d'éléments de preuve ou d'indices pertinents pour la détermination de l'État responsable. Selon un article publié par le site swissinfo le 22 février 2017, « [d]ans l'écrasante majorité des cas, la Suisse a cherché à renvoyer en Italie des migrants qui n'y avaient jamais été enregistrés ». La Suisse spéculerait ainsi sur le fait que les pays qu'elle désigne comme responsables du traitement de la demande d'asile seraient dans l'impossibilité de répondre et seraient par conséquent contraints de (re)prendre en charge les demandeurs d'asile dont elle veut se débarrasser ${ }^{10}$. Il semblerait que la proposition de prise d'empreintes digitales systématique des demandeurs d'asile, clé de voûte du système pour déterminer le pays responsable de la procédure d'asile, soit aussi une invention suisse. Ce qu'expliquent encore Pierre Hazan et Yaël Reinharz Hazan ${ }^{11}$ : « À l'étage inférieur, au bout du couloir, se trouve la salle réservée aux empreintes dactyloscopiques. C'est Arnold Koller, conseiller fédéral en charge de l'asile, qui avait suggéré à ses voisins européens qu'ils prennent exemple sur la Suisse et procèdent systématiquement à la prise des empreintes des requérants. »

Et pour celles et ceux dont le renvoi s'avère presque impossible, la Suisse peut envisager la « disparition ». Là encore, des mesures ont été esquissées dès 1989 et mises en place en 2004 avec l'introduction de l'aide d'urgence pour les déboutés de l'asile. Inscrite dans la Constitution à l'article ${ }^{12}$, elle devrait permettre à « quiconque est dans une situation de détresse et [qui] n'est pas en mesure de subvenir à son entretien d'être aidé et assisté et de recevoir les moyens indispensables pour mener une existence conforme à la dignité humaine ». Or, en réalité, comme le décrit Giada de Coulon dans son ouvrage au titre évocateur, L'illégalité régulière, l'accès à ce droit constitue « un mode de surveillance et même de pression 12 ». Pressions pour que les personnes quittent le territoire ou passent à la clandestinité et ne soient plus un coût pour le pays. Comme le dit sans ambiguïté Kopf, « la "disparition" [des déboutés de l'asile] n'est pas un effet indésirable. Mais la solution que la Suisse a trouvée pour résoudre le problème des renvois impossibles ${ }^{13}$. »

À l'automne 2018, la France a procédé, au mépris du règlement « Dublin », à des réadmissions directes de demandeurs d'asile en provenance d'Italie qui tentaient de franchir sa frontière. Elle s'est appuyée pour ce faire sur un accord bilatéral, l'accord de Chambéry, conclu en 1997 entre les deux pays ${ }^{14}$. Là encore, la Suisse s'était montrée précurseure, puisqu'elle utilisait le même stratagème depuis deux ans $^{15}$. [fin p. 5] 


\section{La chasse aux « mouvements migratoires incontrôlés »}

Le Rapport de 1989, une fois de plus, nous permettra de démontrer la capacité de la Suisse à imaginer, planifier et exécuter sur plusieurs décennies un programme cohérent. Dans ce rapport, apparaissent la notion de « sélection » de la migration et le problème de gestion des « mouvements migratoires incontrôlés ». Il fait ainsi mention des plans helvétiques : «Pour endiguer les mouvements migratoires incontrôlés, il y a lieu de multiplier les efforts en vue de la conclusion d'une convention sur le premier asile et de poursuivre les travaux d'harmonisation du droit d'asile en Europe de même que l'entraide et la coordination administratives. La coopération internationale doit également porter sur la lutte contre les filières de passeurs. "

Ces extraits pourraient ressembler aux recommandations de l'UE pour la décennie à venir... Et la mention de la « convention sur le premier asile » semble anticiper

«Dublin », car ladite convention servirait, dit le Rapport de 1989, à « déterminer à quel État incombe la responsabilité d'une procédure d'asile et [permettrait] d'éviter les mouvements migratoires à l'intérieur de l'Europe ». On y trouve également la note de doléances sur la difficulté à expulser les déboutés de l'asile, problème « résolu » par le passage à la case « rétention », appelée détention administrative en Suisse, avant l'expulsion, notamment pour les « dublinés ». Une mesure entrée en vigueur en France avec la nouvelle loi sur l'asile, mais prévue en Suisse dès 2005. Selon le rapport 2018 de la Commission de gestion du Conseil national, $85 \%$ des 12227 détentions ordonnées en Suisse concernent des personnes " dublinées » et le « taux de succès ", selon les propres mots de la Commission, est remarquable : «Dans $99 \%$ des cas, les personnes mises en détention dans le cadre de la procédure Dublin ont quitté le territoire. [...] En revanche, seules $28 \%$ des personnes NEM Dublin qui n'ont pas été mises en détention ont quitté la Suisse. »

\section{En tête depuis des décennies}

Un récent document interne au SEM et publié dans la presse le confirme : « La Suisse a renvoyé près de $57 \%$ des demandeurs d'asile. Dans l'UE, cette valeur s'élève à $37 \% 16$ ». Pour ce faire, une double stratégie a été mise en place : des adaptations, détaillées plus haut, aux niveaux législatif et administratif, couplées au zèle de la discrète diplomatie helvétique qui a su conclure de nombreux accords de réadmission avec les pays de provenance des migrants.

Si la Suisse, en dépit du succès à l'extérieur de certaines de ses « inventions », n'approche pas un « taux de succès » de $100 \%$, elle se positionne régulièrement en haut du podium des « renvois ». Elle dépasse ainsi d'autres pays européens ; elle laisse surtout face à un mur des personnes en quête de protection, contraintes à la migrerrance sur le continent, à la clandestinité, forcées de retourner vers des pays en guerre ${ }^{17}$ ou d'autres où, dès leur arrivée, elles sont placées directement en détention, comme ce fut le cas pour un ressortissant sri-lankais en $2013^{18}$. La Suisse avait décrété trop rapidement le Sri Lanka « pays sûr ».

Notes: 
${ }^{1}$ Une décision de non-entrée en matière (NEM) désigne la décision des autorités d'écarter un dossier sans examiner les motifs invoqués par la personne requérant l'asile, parce qu'ayant transité par un État membre du règlement « Dublin III », par un État tiers dit « sûr » ou parce que la demande est considérée comme « manifestement infondée ».

${ }^{2}$ Mariana Duarte, Asile à deux vitesses, Observatoire romand du droit d'asile et des étrangers, 2014 : www.odae-romand.ch [en ligne].

${ }^{3}$ Selon le calcul de Vivre Ensemble : https://asile.ch/statistiques/suisse/\#toc7

${ }^{4}$ Le gouvernement suisse a constitué un groupe de travail ad hoc, composé d'experts choisis par trois départements fédéraux (équivalent des ministères français) pour étudier l'évolution des flux migratoires. Il sera mentionné plus bas dans l'article comme « Rapport 1989 ».

${ }^{5}$ Pierre Hazan, Yaël Reinharz Hazan, La Suisse des bons sentiments : voyage en terre d'asile, Les Éditions Metropolis, 1996, p. 108.

${ }^{6}$ Le Temps, 22 juillet 2014.

${ }^{7}$ En Suisse, l'admission provisoire est prononcée lorsque la demande d'asile a été rejetée et une décision de renvoi prise, mais que des motifs juridiques s'opposent à l'exécution de ce renvoi. Pour plus de détails sur ces procédures, voir le site de l'association Vivre Ensemble, Service d'information et de documentation sur le droit d'asile [asile.ch].

${ }^{8}$ Voir notamment l'appel inter-associatif du 18 avril 2018 « contre la levée de l'admission provisoire pour les Érythréennes et les Érythréens » [en ligne :

https: //asile.ch/2018/04/21/osar-erythree-appel-contre-levee-de-ladmissionprovisoire-erythreennes-erythreens $/]$

${ }$ «Factsheet Dublin $»$ :

https://www.sosf.ch/cms/upload/pdf/Factsheet_Dublin_FR.pdf

${ }^{10}$ Selon le règlement « Dublin III » (art. 25), l'État sollicité pour reprendre en charge un demandeur d'asile dispose d'un délai d'un mois (ou quinze jours selon les cas) pour répondre à cette demande. Faute d'avoir répondu à temps, il est réputé avoir accepté la requête et a l'obligation de reprendre en charge la personne concernée.

11 Op. cit.

12 Giada de Coulon, L'illégalité régulière au quotidien. Ethnographie du régime de l'aide d'urgence en Suisse, Éd. Antipodes, 2019, p. 272.

${ }^{13}$ Kopf F., "L'éradication des requérants d'asile déboutés en Suisse », hors-série Vivre Ensemble, 2010, p. 2. [en ligne]

${ }^{14}$ Sara Casella Colombeau, «La frontière définie par les policiers », Plein Droit $\mathrm{n}^{\circ}$ 87, 2010.

15 "The legal frameworks of migrants' rejections from France, Switzerland, Austria », blog Alpine border conflicts. [en ligne] ; Cristina Del Biaggio, « Frontières, migrants et réfugiés : Du bon usage des termes et des chiffres », 2016 [en ligne].

${ }^{16}$ ATS, « La Suisse bat des records en matière de renvois », Le Temps, 21 avril 2019 [en ligne].

${ }_{17}$ RTS, « La Suisse renvoie à nouveau des réfugiés vers des zones de guerre », 21 avril 2019 [en ligne]

18 ODAE romand, « Un Sri lankais passe deux ans en prison après son renvoi : la CourEDH condamne la Suisse », 9 mars 2017 [en ligne]. 